\title{
Green My Place: Evaluation of a Serious Social Online Game Designed to Promote Energy Efficient Behaviour Change
}

\author{
Benjamin Cowley ${ }^{1, *}$, Chris Bateman ${ }^{2,3}$ \\ 1 Corresponding author Cognitive Science, Department of Digital Humanities, University of Helsinki, \\ Finland, ben.cowley@helsinki.fi. \\ 2 University of Bolton, Manchester, United Kingdom, c.bateman@bolton.ac.uk \\ 3 Laguna College of Art and Design, Laguna Beach, CA, United States
}

\begin{abstract}
Serious games are interventions with potential for tackling pressing issues by raising awareness and inciting behaviour change. However, it is unclear which design choices maximise efficient production or intervention efficacy. For example, health games and games tackling social crises may have radically different audiences. Furthermore, players of serious games don't self-select like audiences for entertainment games, suggesting a need to examine and discuss the outcomes of any and all serious games built upon clear design principles for clearly-defined scenarios.

This paper presents a case study of Green My Place, a series game promoting energyefficiency. GMP deployed unique site-specific metrics distinguishing it from similar projects 'disembodied' from the environments they are intended to affect. The game's design methodology - an MMOG framework with atomic mini-games linked to specific learning materials - offers a scaleable generic solution applicable to any domain entailing awareness/education.

Field study evaluations show (weak) positive evidence of a positive impact, but lack of traction hindered success. We examine these outcomes and their possible causes, concluding that although the game itself was a noble failure, the evidence suggests that successful behavioural influence may be independent of degree of engagement - a finding with potential significance for any game with learning objectives.
\end{abstract}

Keywords: serious game, evaluation study, behaviour change, energy efficiency

\section{Introduction}

Serious games have been proposed as a means to tackle even broad societal problems [1], posing a design problem for which the audience model is 'mass-market' and the game platform maximallyscalable. Combining technologies from social networks and intrinsically-motivating games, one compelling new solution is the serious social online game (SSOG) design for promoting behaviour change. SSOGs which may be played in a web browser have very high accessibility; they also have the influencing quality of social norms, and the engaging quality of intrinsically-motivating gameplay experiences. A suitably constructed SSOG encourages problem awareness through immersion in a relevant 'semiotic domain', presenting 'tailored information', both concepts described by Gee [2]. It encourages self-awareness through setting of appropriate goals and giving periodic feedback. It can support the change of values by creation of 'affinity groups', and the 'identity principle' by which individuals can feel part of a larger context whose values can be adopted without conflict with personal values [2]. Finally behaviour change is encouraged when individuals engage with the SSOG system for a sufficient duration, which is several months to one year according to [3].

This paper discusses a specific SSOG, titled Green My Place (GMP), intended to promote changes in energy efficiency behaviour. GMP was designed by the authors and deployed within the European project SAVE ENERGY (CIP-ICT-PSP-238882), which aimed at energy efficient public buildings with five pilots. GMP was built on the requirements and design principles outlined above, 
as elaborated in [4], with a novel implementation tying the general/scalable (social online design) to the specific/personal (pilot site-specific team identities). Specifically, GMP deployed unique sitespecific metrics based on real-time energy-use information from sensors deployed in the pilot buildings, which 'embodies' the game in the environments that its learning-benefits are intended to affect. This paper explores the efficacy of these principles, based on two evaluation trials of GMP, each with separate per-trial populations from five pilot locations.

The two evaluation trials were: a field study among the workers from the five pilots, and a randomised controlled trial testing volunteers recruited from outside the pilots but within the same cities. The field study had the aim of testing players' engagement with the game and with its learning content over a longer term in a naturalistic setting, i.e. without explicit testing and incentives. The controlled trial aimed to test the pedagogical value of the game, measuring data points relevant to problem awareness, self-awareness, change of core values and actual (self-reported) behaviour change; players were given reward incentives to participate.

This serious game project came about in the 2000s as a response to the need to educate citizens towards adopting more sustainable practices, a context where energy usage represents a key issue. Although energy efficiency constantly improves through technological means, the KhazzoomBrookes postulate [5] suggests that such an approach alone can result in the paradoxical increase of total energy consumption (also known as the rebound effect). To obtain a net benefit there must also be efficient usage, implying a responsibility for energy efficient consumption; i.e. minimising waste due to institutional and individual behaviour. While usage can be affected by policy, in most societies policy is in turn affected by individual awareness of the problem and motivation to accept or drive change.

In order to improve individual motivation, awareness and long-term behaviour (with respect to a given issue or cause), evidence suggests a number of pre-requisites, which are more or less causally chained: problem awareness/opportunity $\rightarrow$ self-awareness/capability $\rightarrow$ behaviour and core values/motivation [6]. Within these macro-changes, the Theory of Planned Behaviour suggests that individuals must pass through several stages of change: from asking 'is there a problem?', to 'what is causing it, and what is my part?' and 'do I care?'. Thence to 'what can I do about it?', and 'will it work?'. Finally, perhaps most importantly, many people will also think 'what do my peers think of my behaviour?' [7,8]. This is a lot to change, and therefore requires a powerful intervention; while at the same time the ubiquity of the problem implies the need for an affordable and scalable intervention.

The studies investigating the efficacy of Green My Place broadly support the idea that an SSOG can bring about behaviour change, though with several caveats. Additionally, the discussion elucidates key issues behind the design and implementation of Green My Place that would be applicable to other domains where an SSOG might be considered. This paper should thus be understood as a critical case study of both a project and its design methodology, where the latter can be interpreted as having succeeded despite the marginal successes of this specific project.

\section{Background}

\subsection{Motivation and context}

SAVE ENERGY was a European project that addressed the challenge of behaviour transformation through the use of ICT as an enabler of energy efficiency improvement in five diverse public buildings in five pilots in European cities - Helsinki (two schools), Leiden (city hall), Lisbon (municipality office), Luleå (house of culture) and Manchester (city hall). The project ran from March 2009 to October 2011. The entire project was based upon the European Union's stated goal that public buildings should lead the way in improving energy efficiency [9].

SAVE ENERGY aimed to provide an engaging virtual environment for users, citizens and policy makers to gain awareness, understanding and experience to build energy saving solutions. To this end SAVE ENERGY mainly used ICT tools: the serious game and energy-feedback displays were both connected to real-time energy-use information from sensors and actuators deployed in the buildings. At each pilot SAVE ENERGY had liaisons to coordinate with management and staff, and an energy consultancy firm to implement energy monitoring at selected pilot 'objects' (e.g. individual offices) and to provide the feedback interfaces. GMP was developed as a separate subproject and was promoted in pilots by the liaisons.

At the time the project began, in 2009, the authors had observed that the primary trend in serious game projects was to create simulation games. The operating assumption appeared to be that 
effective behavior change would follow from learning to operate a digital simulation of the subject matter at hand. This assumption was rendered dubious by research on play styles of the audience for games (e.g. [10]) that suggested that detailed simulations were suited to satisfying the play needs of a minority of the population. In targeting a broad demographic audience, a serious game would require a more 'casual' design principle, and at the time of the project beginning there were few models for how this might proceed.

GMP aimed to create a fun and engaging game experience, while shaping the player's behaviour towards energy saving issues. The main focus of game play was to maintain awareness for the user of both energy efficiency issues in general, and exemplary local energy consumption in particular. GMP aimed to achieve behavioural change simply by being in the player's consciousness as much as possible, without impeding their daily routine, and thus keeping the topic of energy efficiency on their mind. Raising the player's consciousness with a long-term 'reinforcement schedule' [11], with low impact on the daily routine but high long-term visibility, was hypothesised to affect their behaviour.

\subsection{Behaviour change in energy efficiency}

In a sense, motivation underpins behaviour. Therefore we cannot expect to see a long-term behaviour change without a steady motivational change sustaining it, and successful interventions need to address motivations as well as attitudes. This seems to be the case for complex behaviours such as energy consumption, where highly personal motivating factors such as attitudes and values play a central role $[3,12,13]$. Motivational interventions have produced some interesting results. Recently, the 'DoNation' project trialled a pledging intervention for eliciting commitments to sustainable behaviour, where the novel component was an ICT toolset [14]. Prior to that, the 'Changing Behaviour' project took an action research approach to model end-user behaviour and stakeholder interaction in energy efficiency (see all publications at http://www.energychange.info/articles, especially $[15,16])$.

The question remains, can the motivations of a large population be affected by an intervention of realistic scale? Stern [17] stressed the plasticity of a population, i.e. the "percent of current nonadopters that could be induced to adopt energy-saving behaviours under acceptable policies". Darby [12] and others have argued that change in behaviour may require proper knowledge of the current situation. They hold that an intervention should lead people to the facts that show the rationality in certain behaviour. Ehrhardt-Martinez [13] added that learning the outcomes of one's actions can lead to long-term behaviour change. Novelty may lead one to try new things every day, but one needs a reason to keep acting in a particular way. Orland et al [18] reported an intervention for energy efficiency in an office, that follows the model of GMP by using real-world data from plug-load sensors to inform participants. Reported energy savings were respectable, although the scale of the intervention was just a single site. Geelen et al [19] also incorporated real energy monitoring in multiple sites and reported good short-term savings, but a strong rebound effect (decreased savings after the game ended). Fell \& Chiu [20] reported that parents were more positive toward energy saving when they consider it as affecting their children's learning, rather than as a financial or environmental concern; this ties in with our use of 'affinity' and 'identity' principles, but poses a problem in public buildings.

A good reason may be necessary, but not sufficient. Other authors (e.g. [3,21,12,22]) have also stressed the importance of feedback, alongside gaining proper knowledge of the subject matter. As noted by Ehrhardt-Martinez [13], social norming may have a central role in behaviour change. According to Ehrhardt-Martinez, the volition of the individual is often not enough to effect change, as the social context needs to support the will to change.

SSOGs are a platform that can offer all the elements of behaviour change stated above: practical information and feedback on one's actions, increased engagement and motivation. The question is, for a specific implementation, whether feeling motivated to play (or to learn through playing) will lead to applying the acquired knowledge to one's daily life — whether one will act. 


\section{Green My Place game design}

\subsection{Structural considerations}

The aims of SAVE ENERGY required a slow burn experience, modelled on massively social online games, or MSO [23], taking the form of short duration educational "energy challenge" mini-games embedded in the ongoing social "Euro Team" meta-game.

GMP itself was played within a WWW-browser (see Figure 1 and design documents ${ }^{1}$; also [4,24]). The five pilot buildings in Helsinki, Leiden, Lisbon, Luleå, and Manchester were each represented as a team in the game. They were motivated through competition to become the most energy efficient over the study duration (for the field study, one year; controlled trial, three months). The energy use monitored by sensors in each building was one source of scoring for each team.

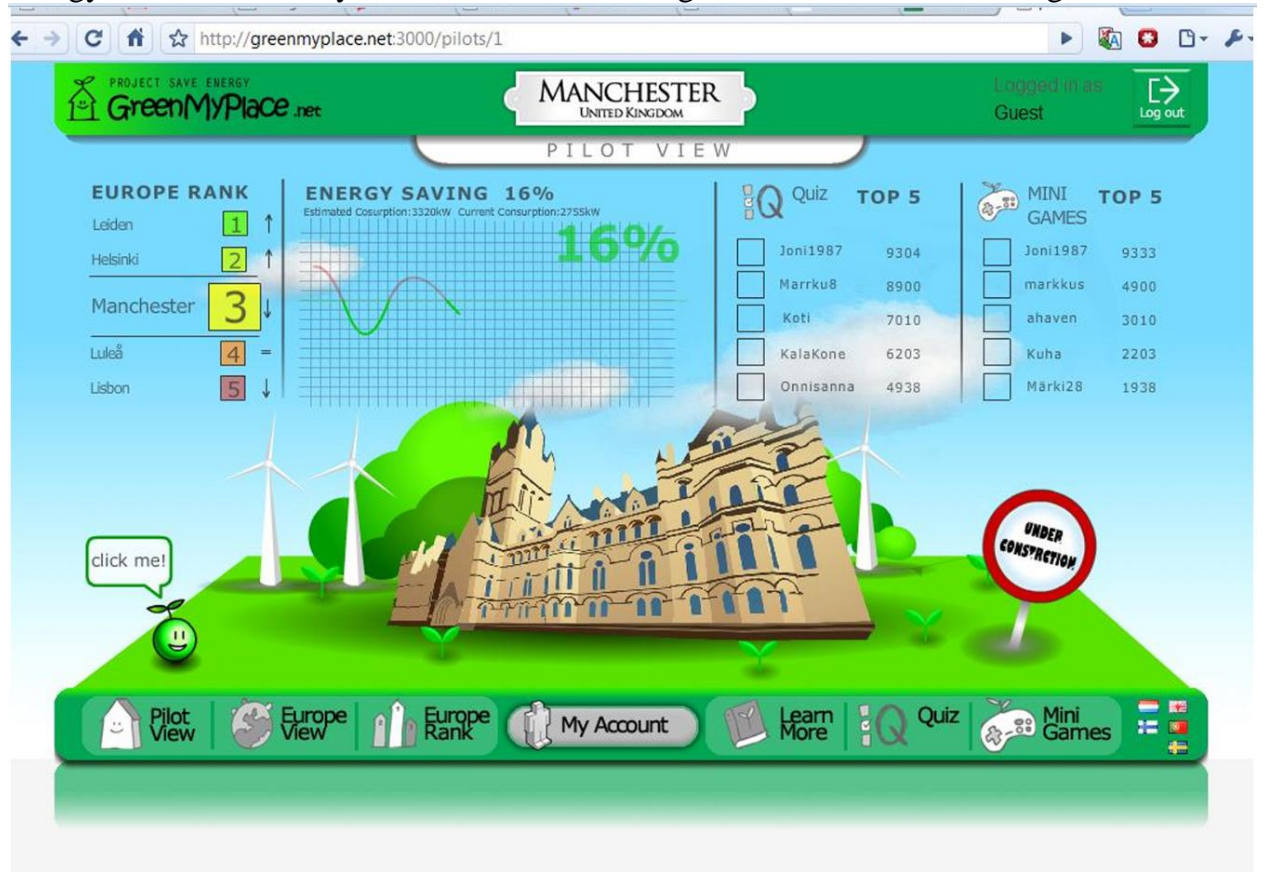

Figure 1. Concept artwork for Green My Place team 'Manchester' page, showing the team rank, Energy Saving data, and unlocked achievements, along with various items around the team building reflecting those achievements, e.g. windmills, plant sprouts.

Additionally, players played simple, fast eco-action based mini-games, took quizzes and learned about energy efficiency from linked sources on the web; this aimed to enhance their awareness and knowledge at the individual level. Such activity contributed to personal and to team scoring. The actions of a team of players combined to win Awards that improve and upgrade the virtual representation of their team building. In this way, the five teams competed for fixed durations to 'green their place'.

The game featured two main types of interaction - direct play as just described, and the social web interaction. In the game's website and other integrated sites like Facebook, the player's score was reflected alongside the score of their chosen pilot building or team. Thus the competition between teams formed a metagame [25] that induced social interaction both within locations, and via social networks. GMP featured a very simple social interface, centred on players' account pages with its record of their activity, but there were insufficient resources to implement a social media platform inside the game. The social interaction was mainly intended to be facilitated through the social spaces of the pilot buildings themselves, and to a lesser degree in the presence of GMP on Facebook.

At the highest level, participation in the game was intended to serve the overall objective to lower energy consumption through behavioural change. Within the metagame, objectives were

${ }^{1}$ Available at https://figshare.com/projects/Green_My_Place/17147 
ranked by timeline: in the short term, one week period, complete one or two available learning tasks or mini-games and maintain energy saving gains. Within mini-games/weekly releases, the objective was to learn a single concept through play. The medium term, one month, objective was to build on the one week achievements to grow a community around the pilot buildings. The long term, one year, objective was to compete with other pilots to become the leading case study for energy savings in Europe. More discussion of the interaction design can be found in [4,24].

Players of the game were ideally expected to experience three principal effects:

1. Improving their awareness of energy efficiency issues (including awareness of one's own inefficient acts and attitudes);

2. leading to an improvement in attitude towards energy efficiency,

3. that in turn leading to improvements in practical acts of energy efficiency.

This process of change was intended to lead to a change in core values, reflected in an increase in scores of universal Schwartz values supporting positive energy efficiency action: Universalism, Benevolence, Security and Self-Direction. However changes in core values do not (and in psychologically-healthy adults should not) happen in a few months, and would require a much more extensive longitudinal study.

In order to evaluate this game we conducted two studies. Over one year the game was released to the inhabitants and users of the pilot buildings in a field study: the game server was open and anyone could join. For three months toward the end of the project a controlled trial, using specially recruited test and control groups, was conducted on an invitation-only server.

\subsection{Mini-games}

Having chosen a format that depended upon mini-games, the design team faced decisions about how to proceed in implementing these. Simulations of energy usage scenarios were discounted for lacking play style diversity, and by budget concerns. What was required were small-scale game activities - mini-games or micro-games, following WarioWare's [26] core design principle - that could then be wed to educational and self-assessment content. The whole package of game-plusassociated-content was thus a wrapper to integrate one or more energy saving behaviours, each one a so-called well-defined 'behavlet' linking resources, actions, and outcomes [4]. With this approach, it was not even necessary (although desirable) that the game itself be educational, since engagement with the game would encourage engagement with other learning materials.

Mini-games were thus the primary mechanic for driving engagement. There are several advantages to simple mini-games over a more complicated single game:

- Simple games are easily learned, and thus can be played by a wide audience

- Simple games can be quickly developed (and thus developed cheaply)

- Different players enjoy different things; having a variety of games on offer greatly increases the chance of any individual player finding a game they enjoy

- Simple games permit easier variation - a simple system can be far more easily altered to fit new data or graphics than a large system of interdependent parts.

Mini-games were available within the main game from an interactive menu called the GreenBox, which was organised as a weekly calendar. A new content was released each week using the GreenBox, with pre-release content visible but locked, as in the example shown in Figure 2.

The mini-games made contributions to the pilot team score in two ways:

1. The Team which had the most number of challenge attempts during each week (i.e. the Team whose members played the mini-games most often) would score points.

2. The Team which scored the highest single result during each week would also score points.

All content was given a visual style or 'skin' to relate it to the pilot building; and because points of this kind were scored weekly, there was a constant yet gentle pressure encouraging supporters to engage in the mini-games, and by extension, to care about what is going on in the pilot scheme which also contributed points into the same pool. A brief discussion of the design of the first five of the mini-games follows (five $=$ four, one of which had two distinct variants). 


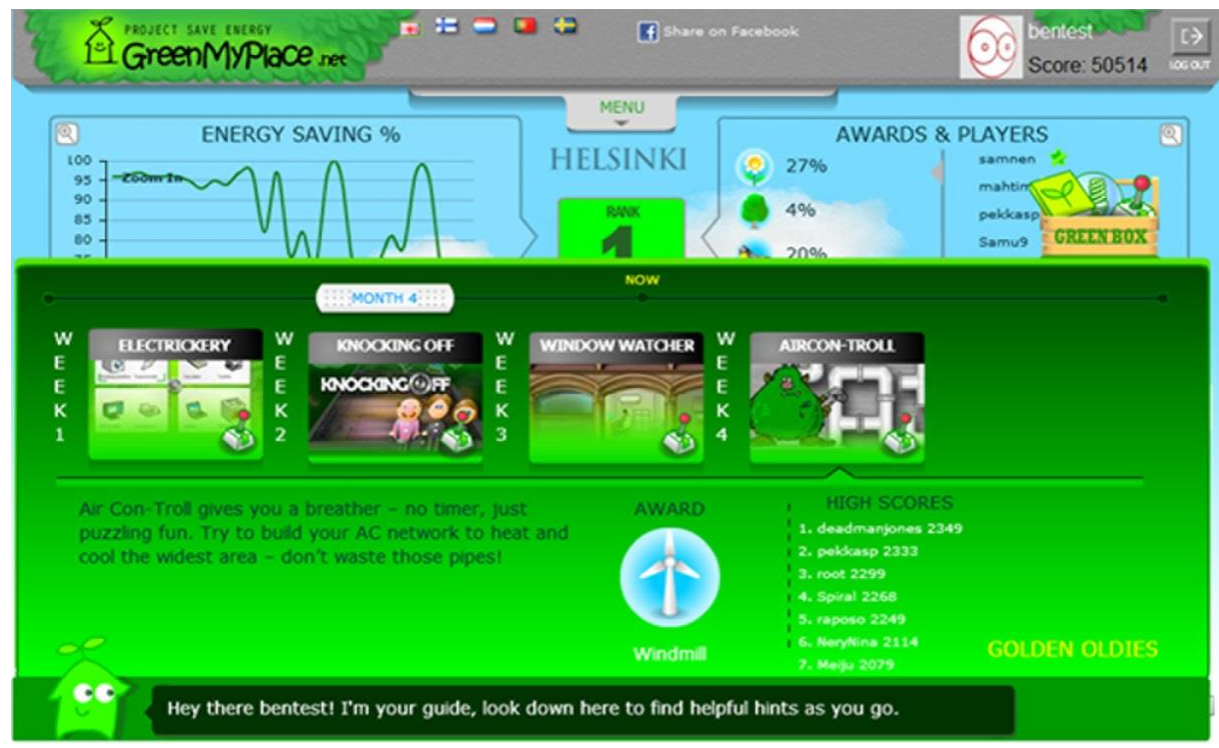

Figure 2. A screenshot of the Helsinki team page with Green Box, showing game options and high score table. Note the player's own overall score and avatar in the top right.

\subsubsection{Window Watcher:}

The concept for this game was to adapt to an energy efficiency context the player practices of a shooting gallery, particularly of the kind that task players with distinguishing between targets and non-targets. The mini-game thus consists of eight windows (modelled on the architecture of the pilot building in each case) showing interior locations such as offices and kitchens. A series of simple animations showed people in the building operating appliances in various ways: the player's task was to spot when something energy inefficient had occurred and click on that window, thus earning points. Examples included leaving lights on when no-one was in the room, not closing a fridge door, and having the air conditioning on while a window was open.

Informally, Window Watcher was one of the best examples in the project of using a classic mini-game design in a manner that was tacitly educational. The design tasks players to analyse what they are seeing with the express goal of spotting energy inefficiency - but it is ambiguous when they start playing what that might consist of. There is no penalty for incorrect clicks, with random clicking a viable (but highly inefficient) scoring tactic for beginning players, allowing newcomers to gradually recognise the energy inefficient situations and thus tacitly acquire (at least in principle) a capacity to recognise the equivalent situations in the building around them.

\subsubsection{Air Con-Troll/Air Con-Troll Puzzle}

This simple mini-game design was modified from the player practices of Pipe Mania [27], with the connection to energy saving being purely cosmetic, and not central to the design. This design had the advantage of being simple to implement, and easy to pick up for players who had encountered a game of this kind before. An additional benefit was producing two variations of the game - one that was puzzle-oriented, requiring a specific solution to found, and one that was closer to the original game's scoring principles.

Although the design principles behind Green My Place allowed for games such as this which had no explicit learning message encoded in the core play, this particular mini-game had limited appeal as well, which rendered it relatively poor in its core task of driving players to engage with the learning materials.

\subsubsection{Knocking Off:}

This mini-game was premised on promoting the idea of turning all the lights off in a building as it is vacated. Cross-breeding player practices from Elevator Action [28] and Lemmings [29], the intent was to have simple quasi-autonomous agents who would move around the building (the Lemmings influence) which would be negotiated by a series of elevators and stairwells (the Elevator Action influence). Regrettably, the resulting game was extremely difficult for players to understand, 
resulting in confusion and relatively low engagement. This illustrates the relative difficulty of successfully 'bolting-on' existing mechanics to fit a given teaching concept.

\subsubsection{Electrickery:}

Both in terms of the views of the design team, and in terms of player uptake, this was the most successful conceptual design. Iterating on the kind of play pioneered by the Brain Training [30] and Big Brain Academy [31] games, Electrickery tasked players to quickly decide which of two sets of appliances used the most energy. Wattage values were only shown after the player had made a choice, requiring players to make intuitive judgements as to which set of electrical devices were the most energy-hungry.

The mini-game was successful both in terms of engaging players, and in having an intrinsic learning element. It generated fairly compulsive play from its fast-paced micro-game decisions, but to succeed players had to develop a strong sense of which of the devices being shown were higher wattage. Because actual approximate power consumption values were used, the educational purposes were delivered elegantly within the core design, and players rapidly got a sense that, for instance, electric fires and microwaves were high energy $(1000 \mathrm{~W}+)$ devices, while computer monitors and refrigerators were relatively modest users of electricity $(<200 \mathrm{~W})$. The more players engaged with the game, the more they learned the wattage values of the appliances in their lives.

\subsection{Learn-More Pages}

In addition to the mini-games, players could also access GMP-pages of content specific to a particular energy-related topic, such as recycling. On these pages there would be links to external online resources, and clicking on these links would generate high scores for the player, such that the player was encouraged to genuinely learn more by an in-game mechanic. Learn More pages were initially topically related to particular mini-games and available only after completing one play of a mini-game. After seeing a positive response to the Learn More format, direct links to custom Learn More pages were posted in the GreenBox as a weekly content (starting in week 12, followed by more games, and another in week 17).

\section{Field study}

The field study aimed to measure as much data as possible about the use of GMP in a natural setting. There were however strict limits imposed by the context: all pilot buildings were managed locations, that is, participants' time was controlled. Workers in municipality offices cannot play games in work time; children in school cannot play games all the time. Additionally, the game was launched halfway through the 2.5 year project, and pilot users had already been exposed to various interventions. Thus there was no chance to perform controlled self-reports and the effects of the game could not be temporally isolated from other interventions. Finally, although GMP was designed as a SSOG, it was deemed inappropriate by stakeholders to obtain any direct measurement of social activity from players in the field study, due to concerns about privacy and/or interruption of work. A solution to this should have been more vigorously sought, but as the problem occurred during development of GMP, the resources of the GMP team were over-stretched.

\subsection{Methods}

Exploratory analyses investigated the GMP players' frequency of playing and their preferences amongst the educational mini-games; their decisions to read more about GMP's educational topics; and third, the relative changes in energy-saving at three comparable test locations.

\subsubsection{Participants}

The participants $(\mathrm{N}=419$; age $28.4 \mathrm{M} ; 9 \mathrm{~min}, 69 \max ; 12.6 \mathrm{std})$ were recruited by targeted advertisements, dissemination events and regular meetings hosted by project partners within each of the five pilot buildings. They were distributed across the pilot cities with number of participants per pilot as shown in Figure 3. Helsinki clearly had the most sign-ups, which can be explained by this pilot being located in two schools; however the number of sign-ups there of age>20 was $\sim 100$, 
suggesting that not all Helsinki players were school children. Overall, the demographic statistics indicate that age is not a barrier to serious game engagement, if deployment is handled correctly.
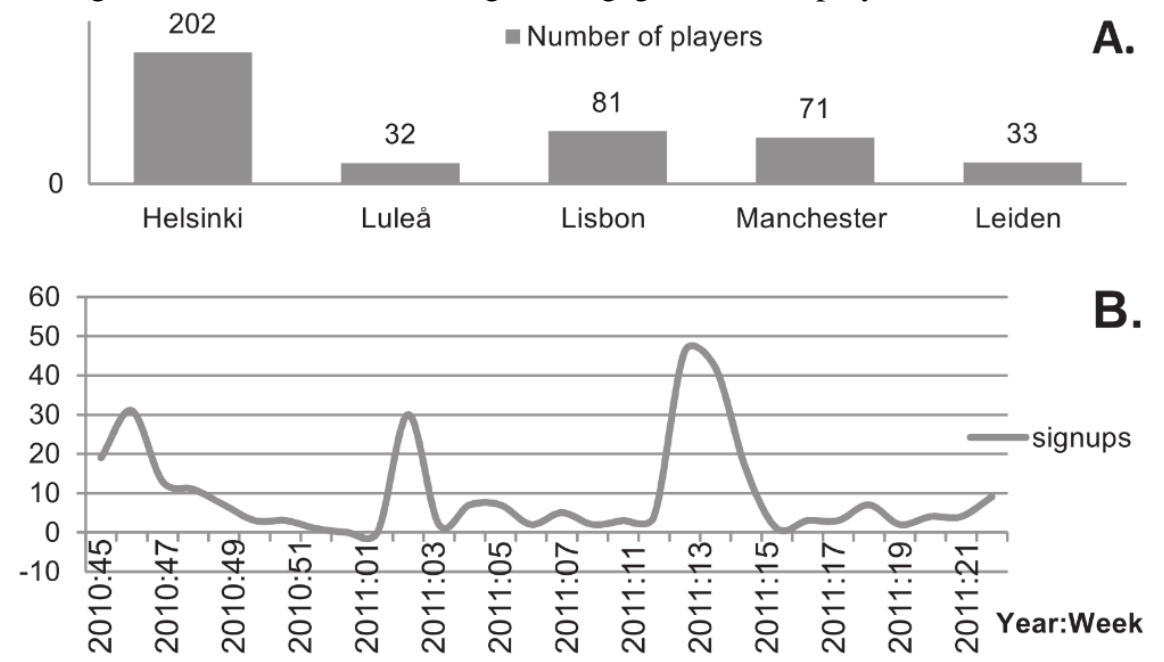

Figure 3. Breakdown of players across pilots in the field study release of the game, panel A. Panel $\mathrm{B}$ shows the rate of signups.

\subsubsection{Apparatus and Materials}

The mini-games, Learn More pages, and other interactive design of the GMP main release are described above. Energy consumption data was read into the game via a central server which collated the output from energy monitoring equipment installed within each pilot building. The implementation details for all pilots are given in documentation of the SAVE ENERGY project ${ }^{2}$.

Analysis of energy-use patterns for this field study is focused on Luleå 'Kulturens Hus' pilot building, because its test 'units' present the only naturally occurring pseudo-test and -control units within the project ${ }^{3}$. Other pilots had more homogenous test units (Lisbon, Leiden: all offices; Helsinki: all classrooms; Manchester, café). Luleå test units were three separate locations: a music hall used for meetings, rehearsals and performances; offices of the Kulturens Hus staff, centred around a common meeting/coffee room area; and the kitchens of the Kulturens Hus restaurant. In each Luleå test unit, energy measurement was enabled (with a Saber device from KYAB Sweden $\mathrm{AB}$ ) and displayed on real-time information (RTI) display screens (which also displayed Luleå's GMP status). In each test unit, staff had usual meetings with project liaisons and technicians. However, the office is differentiated from the music hall and restaurant because office staff also had access to actually play GMP via their work PCs, in their spare time.

Thus, each Luleå test unit had greatly different usage patterns in terms of staff behaviour and energy consumption, but all share the same intra-pilot interventions, except access to GMP. Thus, the Luleå 'test objects' provide a self-contained test of the effect of GMP in addition to the pilotspecific interventions.

We chose not to compare energy consumption directly between pilots because their locations, test objects and measurement equipment were all independent and rather different. That was a deliberate design choice of the project, in order to facilitate the most efficient technical solution in each location, and was not under the influence of the GMP design team.

\subsubsection{Procedures}

Participants played the main release of the game, an open server where they could sign up to one of the pilot building teams, and gain access to Mini-games and Learn More pages. We collected user behaviour data from the GMP server logfiles to report on the Mini-games and Learn More Pages. All activity of interest within GMP was logged by server-side scripts to a MySQL database, from where it could be mined to discover patterns of player behaviour. For example, visits to mini-games, completed mini-games, etc.

\footnotetext{
${ }^{2}$ Available at https://figshare.com/projects/Green_My_Place/17147

${ }^{3}$ We say 'pseudo' because these were not designed as a set of test and control units, but arose emergently.
} 


\subsubsection{The Mini-games}

We mainly characterise the mini-games by patterns of high-level interaction; Playing the minigames was, however, entirely optional for the user. To study the interest towards these interactive learning modules, we used the GMP server's records of mini-game page visits (logged whenever a player clicked on the mini-game icon in the GreenBox) and completed mini-game plays (logged whenever a mini-game sent a score to the backend at the end of a game).

From these data we compared the number of visits, completed plays, and game scores, thus forming an estimate of the revisit/replay value of each game. All games had been released for at least three months by the date of these calculations. As the release dates still varied, however, we normalised the play counts by the games' "age" before their mutual comparison, and calculated the possible effects of novelty through a correlation test between user signups and mini-game page visits.

\subsubsection{The Learn More Pages}

While the amount of play sessions is a fairly reliable measure of interest towards the games, it does not give information about whether the user was interested in the game's educational content (i.e. ways of saving energy). We assumed that users who were interested in the subject matter of GMP would mainly be the ones visiting the Learn More pages, acquired from server's records of Learn More page visits.

\subsubsection{Energy Consumption}

In all five pilot buildings, saving energy was promoted with energy usage screens in public areas and GMP (wherever users could play). SAVE ENERGY measured the (change in) energy consumption followed by these activities, using the International Performance Measurement and Verification Protocol (IPMVP®). In the pilot intervention test methodology, IPMVP option $\mathrm{B}^{4}$ was used:

$$
\text { Savings }=\text { Baseline Energy }- \text { Reporting Period Energy }+/ \text { - Correction Factor }
$$

Technicians validated the setup by comparing historical values with test data established during a 6 month reference period. During the same period the project established the correction factors to use. This was done by comparing actual energy consumption with the assumed correction factor, validated with linear regression $\mathrm{R}^{2} \geq 0.9$.

\subsection{Results}

\subsubsection{The Mini-games}

The results show that all five mini-games succeeded in attracting users. In Figure 4 we see the normalised play statistics for each of the first five mini-games released. Panel A shows the (normalised) number of visits for the first five games released. Shown alongside is the number of (distinct) visits of a unique player - that is, discounting repeat visits, so that the number of players in light grey columns equals that in dark grey columns. Panel B shows the number of completed plays, again contrasted with the number of unique completed plays. We can see that all games had a similar re-visit ratio - i.e. the ratio between visits and unique visitors (Panel A). Electrickery was the most visited, and the most played along with Window Watcher (Panel B). The play to unique player ratio in these two is notably larger, meaning that players who played through to the end played a relatively large number of games.

As shown in Figure 4, Electrickery had a similar number of visits (170) and plays (163), meaning almost everyone who clicked, also played and finished (logged a score). Window Watcher had a higher number of plays (133) than visits (106), meaning that some visitors completed more than one game per visit by hitting the 'replay' button - indicating a high replay value. The last three games show much less completed plays than visits: Aircon-Troll Puzzle (visit 122, play 16), AirconTroll (visit 135, play 34), Knocking Off (visit 140, play 36). However, these games still persuaded

\footnotetext{
${ }^{4}$ IPMVP is trademark of Efficiency Valuation Organization. See http://www.evo-world.org
} 
some players to play through, multiple times, since completed plays is greater than distinct players - indiciating that these games may have had a more niche appeal than the first two games, perhaps because they were puzzle-style games.

Finally we can look at the aggregate values for visits and scores across all these five games in Figure 5. There is a low overall average for both visits and scores, with some spiking activity at three points. Comparing the mini-game visit/mini-game score spikes with signup data, we found that these spikes correlate with the signups $(r=0.89, p<0.05)$. This implies that the novelty of the GMP platform as a whole drove most of the activity in relation to the games.

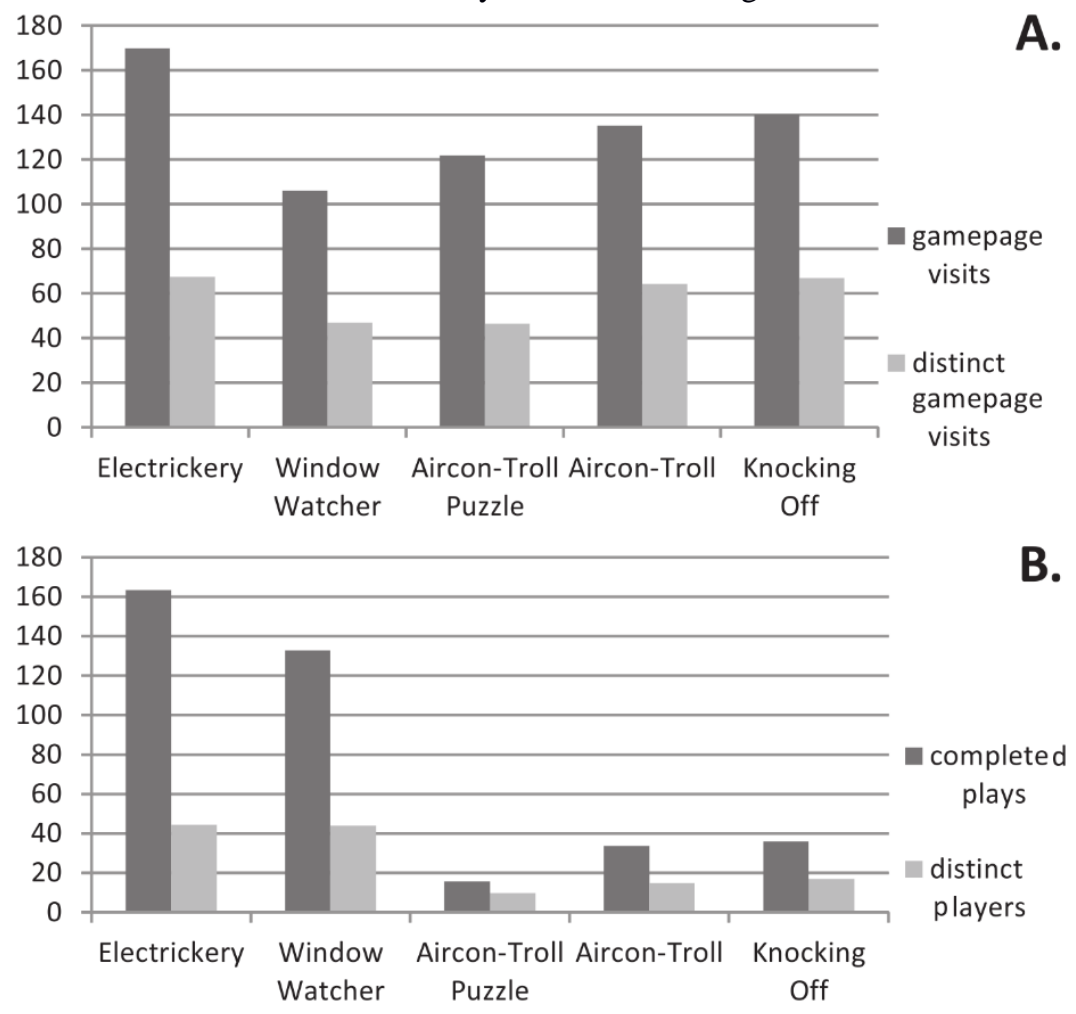

Figure 4. Mini-game interactions.

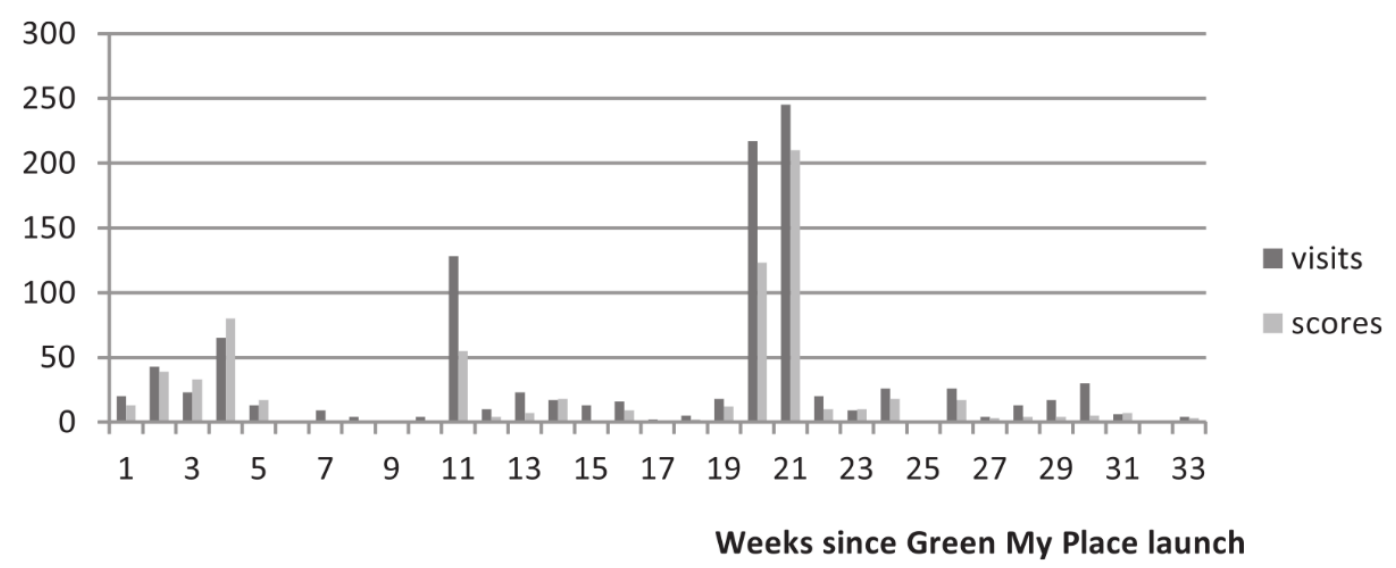

Figure 5. Mini-game visits = dark grey bars; against mini-game scores= light grey bars. 


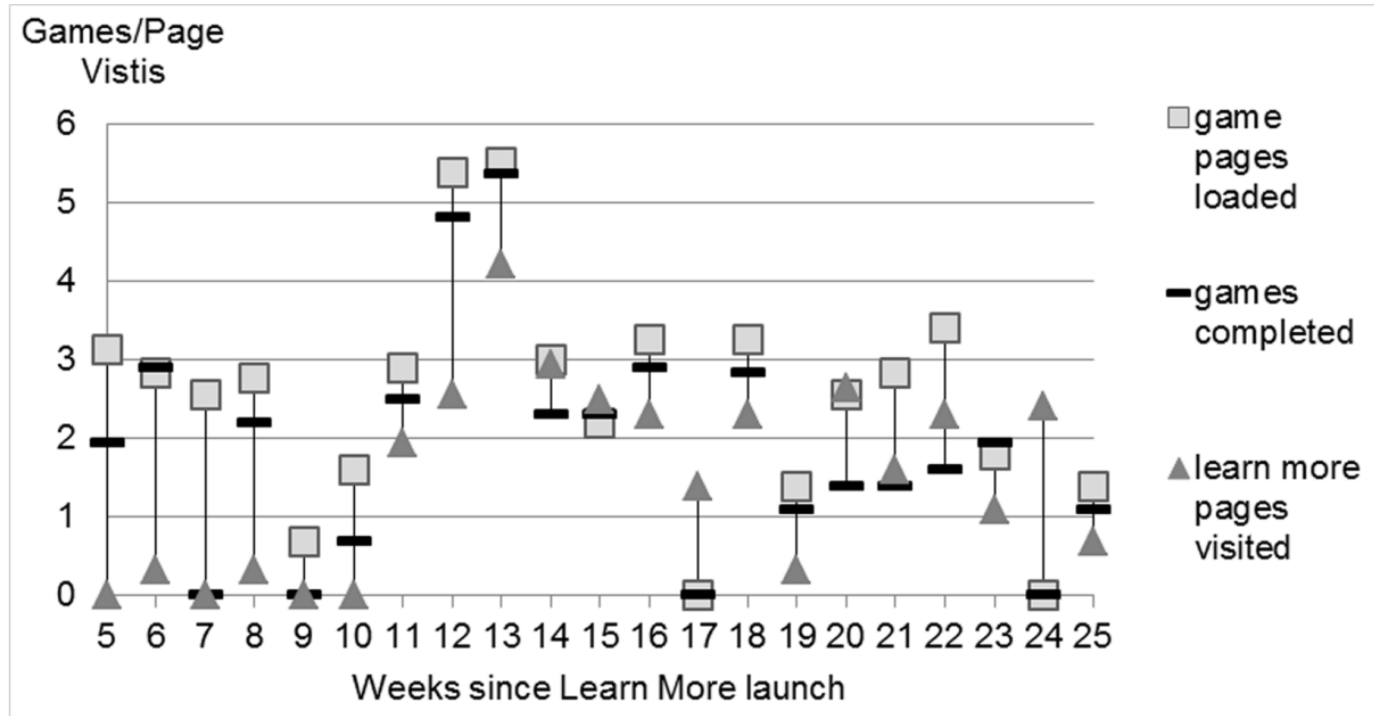

Figure 6. Rate of Learn More visits compared to games played (log-transformed), listed in terms of calendar weeks during 2011.

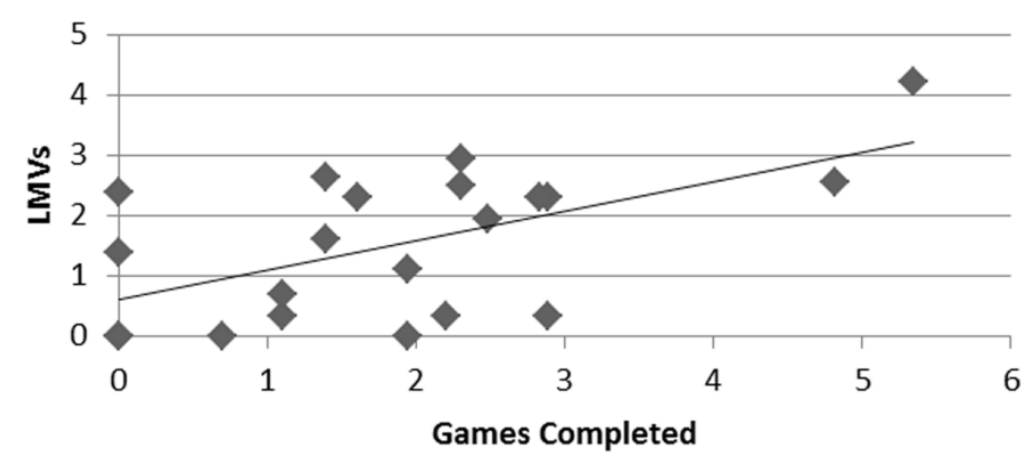

Figure 7. Plot of Learn More page visits (LMVs) against games completed (natural log scale for ease of viewing).

\subsubsection{The Learn More Pages}

The number of Learn More page visits (LMVs) varied throughout the followed period, with a major increase around week 11 corresponding to an upturn in player numbers (see Figure 6 - all values are $\log$ transformed to be comparable in display). Since in every week only one activity is released and after week 11 there were some direct links to custom Learn More pages posted in the GreenBox, thereafter some weeks even show high LMVs combined with zero game activity.

Comparing LMVs in the weeks of zero game activity against others we found an increase of $23 \%$ when games are played. Further, we found that more LMVs occurred without games completed than vice versa. There exists a strong correlation between games completed and LMVs, which we calculate as $r=0.58, F(1,19)=9,6, p<.01$ (see Figure 7).

\subsubsection{Energy Consumption}

Comparison of the three test objects at Luleå (office, music hall and restaurant) revealed that the restaurant was the greatest consumer of energy, while the music hall's consumption varied most. In relative terms (highest savings value proportionate to consumption), the greatest saver was the office. In terms of meeting the baseline target, the office was also the most consistent.

Energy savings from these test objects fell within the range 7-30\%. These energy savings are fully a result of the User Behaviour Change. The results per test object, in terms of annual savings 
percentage and kilowatt hours $(\%, \mathrm{kWh})$ were: restaurant $(8 \%, 17708 \mathrm{kWh})$, music hall $(7 \%, 1470$ $\mathrm{kWh})$ and office $(30 \%, 15260 \mathrm{kWh})$.

\section{Controlled trial}

The controlled trial addressed questions which the field study could not, namely between-groups comparison of GMP's psychological effects. The controlled trial was itself limited in the sense that the participants could not be pilot building workers or users, and thus energy measurements from the pilot buildings could not be analysed even to the limited extent of the field study. Further, motivation to play was manipulated by the incentives used for recruitment, therefore direct analysis of the game activity is not valid. The controlled trial thus restricts analysis to self-reports (controlling for players' quantity of activity)

\subsection{Methods}

The controlled trial evaluation aimed to discover whether engaging with GMP would improve attitudes, knowledge or behaviours in respect to energy efficiency, by comparing a randomly selected population of players against a control group of non-players. Our expected effects are formalized as the following hypotheses:

A. HA-action: Compared to not playing, using GMP would result in an improvement in selfreported practical acts of energy efficient behaviour.

B. HB-attitude: Where energy efficient behaviour did not improve, compared to not playing, using GMP would result in improved self-reported attitudes towards energy efficient behaviour.

C. HC-awareness: Where energy efficient attitudes did not improve, compared to not playing, using GMP would result in improved self-reported awareness on energy efficient behaviour, including awareness of one's own inefficient acts and attitudes.

We also wanted to control for the impact of game engagement, that is, how much players actually were involved in the game. For this we used a measure of how often the player visited each part of the meta-game site including the mini-game pages. With a median split to distinguish high from low participation game players, we can compare the following combinations: all players (ALL) vs. control (CTRL), high participation players (HI) vs. control, low participation players (LO) vs. control and high participation vs. low participation. The last combination doesn't address the above hypotheses (doesn't involve controls), so we propose the following final hypothesis:

A. HD-engagement: The level of active participation in the game would be reflected in the outcome variables measuring participant behaviour, motivation, awareness and values.

\subsubsection{Participants}

In accordance with the declaration of Helsinki, participants were thoroughly briefed on the purpose and procedure of the study; each signed a written informed consent prior to the experiment. Participants were also reminded that they could withdraw from the study at any time without fearing negative consequences. As the study did not concern medical research, it required, in accordance with Finnish law, no formal ethical approval from the Ethics Review Board of Aalto University.

130 participants were recruited from all pilot cities (see Table 1, columns 1, 2). 51 participants dropped out. The remaining 79 participants were randomly assigned to test and control groups, with roughly three quarters in the test group and one quarter in the control group (Table 1, columns 3, 5). There were participants in all pilots, however, the majority were recruited in Helsinki due to better resources for managing contact (i.e. this was where the experiment team was based). We recorded pre-and post-test universal Schwartz values for reference; but they did not change and are not reported. The distribution of background variables was not significantly different between test and control group, including: age, gender, baseline universal Schwarz values. 
Table 1. Participant breakdown by pilot and test-control groups, with age data.

\begin{tabular}{l|l|l|l|l|l}
\hline Pilot & $\begin{array}{l}\text { Local n } \\
(\mathbf{f} / \mathbf{m})\end{array}$ & Test (f/m) & $\begin{array}{l}\text { Test age: } \mathbf{M}, \\
\text { SD }\end{array}$ & $\begin{array}{l}\text { Control } \\
(\mathbf{f} / \mathbf{m})\end{array}$ & Control age: M, SD \\
\hline Helsinki & $70(51 / 19)$ & $45(32 / 13)$ & $26,5.4$ & $12(10 / 2)$ & $26,3.3$ \\
\hline Leiden & $4(1 / 3)$ & - & - & - & - \\
\hline Lisbon & $22(11 / 11)$ & $5(4 / 1)$ & $32,8.5$ & $3(2 / 1)$ & 33,5 \\
\hline Luleå & $12(3 / 9)$ & $1(0 / 1)$ & $33,-$ & - & - \\
\hline Manchester & $22(15 / 7)$ & $11(8 / 3)$ & $37,10.7$ & $2(2 / 0)$ & $46,4.9$ \\
\hline Total N & $130(81 / 49)$ & $62(44 / 18)$ & & $17(14 / 3)$ & \\
\hline
\end{tabular}

\subsubsection{Experiment Design}

The protocol is detailed schematically in Figure 8 below. A pre-test and multiple post-tests were included to track trends. The tests were performed for both groups (test, control) in parallel, simultaneously in all test cities. The test players played the games for a fixed three-month period on a rebooted closed-server version of GMP with the goal of outperforming the rival teams.

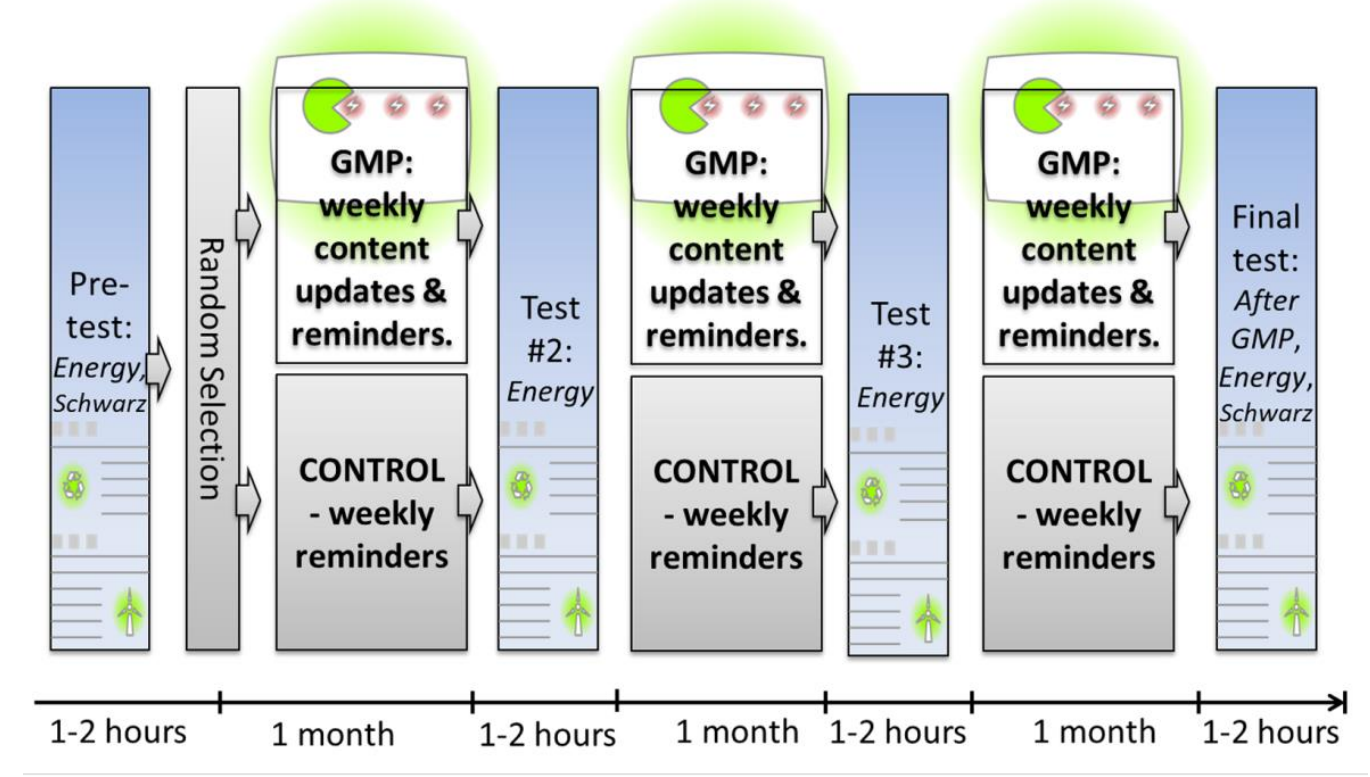

Figure 8. Experimental protocol of the controlled trial; players' track is above, control group below.

As in the field study's open-server release, all activity content was presented through a 'Green Box' weekly activity mechanism. Unlike the open server, once an activity was released in the controlled trial an email message was sent to each participant announcing the activity's release. The extent of participants' play after that was dependent on their motivation. For the control group, a message was sent with a short text encouraging energy-efficiency, to match the degree of experimenter contact with test group.

\subsubsection{Tests and Materials:}

The experiment relied on several measurement instruments, to establish baselines and subsequent change in subjects' attitudes, motivation, and energy use practices over the course of testing, see Figure 9. The pretest contained the background information, Energy and Schwartz questionnaires; tests 2 and 3 contained only the Energy questionnaire; and the final test contained the After GMP, 
Energy, and Schwartz questionnaires5. For details of these questionnaires see supplementary material. The development of the questionnaires and their application protocol was inspired by Schultz's work on the structure of environmental concern [32]; the behaviour change wheel [6]; Schwartz's model of values [33]; and reviews of behaviour change interventions [3].

A background information questionnaire studied the participants' level of experience with computers and serious gaming, current housing situation and whether they had any dependents.

The Energy Questionnaire was designed to answer hypotheses HA-action, HB-attitude, HCawareness and HD-engagement with sections to assess three themes: player awareness, motivation and practical energy-use behaviour. Each theme included a number of factors based on multiple questions. These thematic factors form the primary measure of change in each player as they experienced the game.

The progression of population-mean scores in the Energy Questionnaire across four test times was found to be monotonic, therefore we concentrated only on difference between pretest and final test. Behavioural and awareness factors in the Energy Questionnaire are measured by the difference scores from test to test: if answer 2 minus answer 1 is positive, that is a trend of improvement. For motivation (of the self and other) factors, we take the difference between self and other factors. If the participant rates a motivating factor higher for themselves than for others, this is a sign of social responsibility, of rejecting the tendency to attribute blame to others. The 'improvement' score for all factors for each participant in the test and control groups constituted our dependent variable in statistical analysis. Independent variables were the group which participants belonged to. Thus we tested our four groups, ALL vs. CTRL, HI vs. CTRL, LO vs. CTRL and HI vs. LO, for difference of means using the t-test.

The After GMP Questionnaire asked players to rate GMP on a 4-point Likert scale, describing:

- how well designed was the interface,

- if they enjoyed playing the game,

- if they could navigate and obtain sufficient help functions,

- if they were motivated by the game (more on that below),

- if the game represented a valid training tool for energy efficient behaviours,

- if they gained worthwhile knowledge from playing, and

- if the game had any impact on their thinking or behaviour overall.

We report results as the percentage of respondents who gave positive (i.e. above the mean) responses. The players also answered open questions about how they had changed their energy consumption behaviour after first starting to play. This part was meant for qualitative game impact evaluation and for its further development, and did not answer any particular hypothesis.

\subsection{Results}

\subsubsection{Hypothesis A - Action}

We found support for HA-action in Behaviour, where players were significantly different to controls, by one-tailed tests. Table 2 displays those results which were significant at $p<0.05$; all of medium effect size $\geq 0.3$.

Table 2. Results supporting hypothesis HA-action, where players' scores for Behavioural factors were significantly better than control.

\begin{tabular}{l|l|l|l|l|l|l|l}
\hline COMBINATION & FACTOR & $\begin{array}{l}\text { Mean } \\
\text { Players }\end{array}$ & $\begin{array}{l}\text { Mean } \\
\text { Control }\end{array}$ & $\begin{array}{l}\text { DIFF } \\
\text { SCORE }\end{array}$ & dF & $\begin{array}{l}\text { t- } \\
\text { Stat }\end{array}$ & $\begin{array}{l}\text { Effect } \\
\text { size }\end{array}$ \\
\hline ALL vs CTRL & BATHROOM & 0.038 & -0.443 & 0.48 & 72 & 3.16 & 0.35 \\
\hline ALL vs CTRL & RECYCLING & -0.015 & -0.382 & 0.37 & 72 & 2.62 & 0.295 \\
\hline HI vs CTRL & BATHROOM & 0.026 & -0.443 & 0.47 & 44 & 2.36 & 0.336 \\
\hline LO vs CTRL & BATHROOM & 0.051 & -0.443 & 0.49 & 43 & 2.46 & 0.351 \\
\hline LO vs CTRL & RECYCLING & 0.043 & -0.382 & 0.43 & 43 & 2.26 & 0.326 \\
\hline
\end{tabular}

${ }^{5}$ The 'Human Values Scale' in Dutch, English, Finnish, Portuguese and Swedish was obtained from the European Social Survey website. See http://www.europeansocialsurvey.org/data/themes.html?t=values 
However, other evidence was found against HA-action. Table 3 displays the counter-evidence results significant at $p<0.05$; only Heating $\mathrm{A} / \mathrm{C}$ results had medium effect size. Out of eight factors for Behaviour three are convincingly significantly different, two supporting and one contrary to HAaction.

Table 3. Results contrary to hypothesis HA-action, where control scores for Behavioural factors were significantly better than players'. A/C stands for air-conditioning.

\begin{tabular}{l|l|l|l|l|l|l|l}
\hline COMBINATION & FACTOR & $\begin{array}{l}\text { Mean } \\
\text { Players }\end{array}$ & $\begin{array}{l}\text { Mean } \\
\text { Control }\end{array}$ & $\begin{array}{l}\text { DIFF } \\
\text { SCORE }\end{array}$ & dF & t-Stat & $\begin{array}{l}\text { Effect } \\
\text { size }\end{array}$ \\
\hline ALL vs CTRL & LIGHTS & -0.022 & 0.259 & -0.28 & 72 & -2.12 & 0.24 \\
\hline ALL vs CTRL & HEATING A/C & -0.023 & 0.623 & -0.65 & 72 & -4.75 & 0.49 \\
\hline HI vs CTRL & HEATING A/C & -0.06 & 0.623 & -0.68 & 44 & -3.82 & 0.5 \\
\hline LO vs CTRL & HEATING A/C & 0.015 & 0.623 & -0.6 & 43 & -3.39 & 0.46 \\
\hline
\end{tabular}

\subsubsection{Hypothesis B - Attitude/Motivation}

The attitude of participants was measured by the motivation factors, which are split in two sets. One set asked whether anything made it impractical to save energy, and showed no significant betweengroups differences. Table 4 shows the evidence we found for and against hypothesis $\mathrm{HB}$-attitude: positive or negative values in the DIFF SCORE shows whether it is supportive or contrary evidence. This theme provides an inconclusive case.

Table 4. Results related to hypothesis HB-attitude, i.e. where the players differed from the control group.

\begin{tabular}{l|l|l|l|l|l|l|l}
\hline COMBINATION & FACTOR & $\begin{array}{l}\text { Mean } \\
\text { Players }\end{array}$ & $\begin{array}{l}\text { Mean } \\
\text { Control }\end{array}$ & $\begin{array}{l}\text { DIFF } \\
\text { SCORE }\end{array}$ & dF & t-Stat & $\begin{array}{l}\text { Effect } \\
\text { size }\end{array}$ \\
\hline ALL vs CTRL & RATIONAL & -0.002 & 0.222 & -0.223 & 72 & -4.09 & 0.43 \\
\hline ALL vs CTRL & ECOLOGICAL & -0.009 & 0.105 & -0.114 & 72 & -1.84 & 0.21 \\
\hline ALL vs CTRL & JUSTICE & 0.003 & -0.092 & 0.095 & 72 & 2.24 & 0.26 \\
\hline HI vs CTRL & RATIONAL & -0.025 & 0.222 & -0.25 & 44 & -3.7 & 0.49 \\
\hline LO vs CTRL & RATIONAL & 0.022 & 0.222 & -0.19 & 43 & -2.9 & 0.41 \\
\hline LO vs CTRL & JUSTICE & 0.03 & -0.092 & 0.12 & 43 & 2.65 & 0.38 \\
\hline
\end{tabular}

\subsubsection{Hypothesis C - Awareness}

In Table 5 we present results where $\mathrm{p}<0.05$ for Awareness factors; effect size is again medium or below. Once again, we cannot easily distinguish players from control group. There is clearly some kind of difference based on the number of significant results, suggesting that we can reject the null hypothesis, but the nature of the difference remains unclear.

Table 5. Results related to hypothesis HC-awareness from the Energy questionnaire.

\begin{tabular}{l|l|l|l|l|l|l|l}
\hline COMBINATION & FACTOR & $\begin{array}{l}\text { Mean } \\
\text { Players }\end{array}$ & $\begin{array}{l}\text { Mean } \\
\text { Control }\end{array}$ & $\begin{array}{l}\text { DIFF } \\
\text { SCORE }\end{array}$ & dF & t-Stat & $\begin{array}{l}\text { Effect } \\
\text { size }\end{array}$ \\
\hline ALL vs CTRL & $\begin{array}{l}\text { ELECTRICITY } \\
\text { BILL }\end{array}$ & -0.192 & 0.188 & -0.380 & 72 & -1.998 & 0.229 \\
\hline ALL vs CTRL & KITCHEN & -0.027 & 0.770 & -0.797 & 72 & -2.789 & 0.312 \\
\hline ALL vs CTRL & WORK & 0.260 & -0.901 & 1.161 & 72 & 2.671 & 0.300 \\
\hline ALL vs CTRL & $\begin{array}{l}\text { Public vs } \\
\text { residential... }\end{array}$ & 0.018 & -0.588 & 0.606 & 72 & 2.685 & 0.302 \\
\hline
\end{tabular}

While a particular group of factors were recorded to measure the theme of Awareness, it is also possible that large effects in Behaviour and Motivation, whether positive or negative, could represent 
the effect of the game on their awareness. Consider Behaviour, for example: If a player first says that they do a lot of energy saving actions, but later realises that they do far less than they first maintained, we would obtain a large negative difference score - but if the score was significantly different to the control group it would still be a sign that playing the game had an Awareness effect.

To measure this case we look for an undirected difference between test and control group, by running a two tailed test. In the case of Behaviour, the results were significant for both one- and two-tails. Thus we can simply look again at Table 2 and Table 3, which show us that the mean difference score for the player group is an order of magnitude smaller than that of the control group, both for factors which support HA-action and for against it. In the results from Table 4, the factor ECOLOGICAL was not significant in a two-tailed test, but the factors RATIONAL and JUSTICE were. Again, the mean difference scores from each group give evidence against $\mathrm{HC}$-awareness.

\subsubsection{Hypothesis D - HIGH versus LOW engagement:}

As can be seen from results for previous hypotheses, there was very little difference between players of high and low levels of participation. This is even more surprising given that the low participation players completed on average less than 3 gameplays. Thus, we can conclude that serious games' efficacy does not depend on high commitment from the players, and this represents a potentially important finding of this study. In the context of serious games that aim to influence attitudes and behaviors, the idea that a relatively short engagement could still provoke significant change provides reasons for games of this kind to focus on compact, readily-encountered player experiences rather than complex, in-depth systems play. This aligns with the motivations of the development team in undertaking an SSOG, but it also suggests that even more compact designs might be viable promoters of change, especially in cultures where smartphones increasingly dominate the recreational use of electronics.

\subsection{5 'After Green My Place' Questionnaire:}

Questionnaire 2, 'After Green My Place', which was given to players of the controlled test group after the study had finished, returned some valuable results. Overall GMP was experienced in a positive manner as can be seen from Table 6 .

Table 6. Factors from the After GMP questionnaire, rated by test players. Right-hand column is a percentage of positive responses on a 4 point Likert scale (i.e. above the mean).

\begin{tabular}{l|l|}
\hline Rating Factor & Positive response \\
\hline Interface & $58 \%$ \\
\hline Playing - Storyline and Design & $64 \%$ \\
\hline Navigation/Help & $61 \%$ \\
\hline Enjoyment/motivation by playing the game & $60 \%$ \\
\hline Training & $63 \%$ \\
\hline Knowledge gained & $56 \%$ \\
\hline Impact & $61 \%$ \\
\hline
\end{tabular}

Players also answered some open questions on the effect of the game: the percentage of those who responded positively is given alongside the wording below:

1) "Since playing the GMP have you started checking the energy consumption ( $k W)$ figures of electrical appliances at home or work?" - $32 \%$ yes.

2) "Since playing the GMP have you started limiting the use of electrical appliances that consume relatively high amounts of electricity?" - $48 \%$ yes.

3) "Has playing the GMP game changed your views on what it takes for people to start saving energy? If yes, how?" - $23 \%$ yes, including:

a) "The game has reminded that people have a tendency to easy solutions and to the narrative nature of entertainment such as games - these could also be used in energy saving campaigns."

b) "I have become more aware of the importance of the attitudes of the close ones (friends, family, working environment) and their impact. It is bigger than I would have thought beforehand."

c) "I have understood that the social pressure might have a bigger role than expected."

d) "I know better now which electric appliances waste energy the most." 
e) "Playing the game has made me think that I should campaign and encourage others to save energy as I do, because social pressure is the best tool for implementing change."

4) "If you have adopted new energy saving actions after playing the GMP, has the game played a role in it, and how?" - $29 \%$ yes, and some responses included:

a) "I didn't know that some of the electric appliances waste so much energy."

b) "I use nowadays e.g. the residual heat of the oven and stove, which I hadn't done before."

c) "I now favour energy efficient light bulbs."

d) "Switching off standby. Monitoring energy consumption. Will consider energy consumption before purchasing new items."

\section{Discussion}

GMP successfully engaged with players and brought many of them into a new relationship with energy efficiency, even though statistical analysis of the controlled-study hypotheses were relatively inconclusive. In the controlled study, in all categories where there was measurable change in the players versus controls, there was improvement in some subcategories yet decline in others. Some of the ambiguities follow from the interaction between questionnaires and the impact of the intervention on subjects' self-evaluation. This is because subjects, being situated humans, would tend to change their self-evaluation of ecological behavior when given factual knowledge about environmental issues. People who thought they behaved environmentally soundly learned during the study that they were not doing so optimally; this could cause their responses in later questionnaires to decline, even if their actual behavior might have improved.

Thus, for hypotheses $A-C$ we have the situation that the evidence partially suggests a difference between test and control groups, mainly supportive of a change in the energy saving behaviour of players (hypothesis $A$ ); but it feels inconclusive. This outcome is not ideal, however given the responses to questionnaire 1 'After GMP' and given that a difference clearly exists, it seems safe to assume that the game does positively affect players' relationship with energy efficiency. Here it is important to note that we did not start from nowhere: many controlled-study participants in the pilot cities were already being made at least somewhat environmentally aware, by SAVE ENERGY and other projects. However, they might lack knowledge about efficient ways to turn that motivation into actions. This lack of knowledge could result in just the kind of pattern seen in the study: more change in hypothesis $A$ than in $C$ and especially $B$, reflecting already-motivated players who were learning concrete ways to save energy through the game.

From Hypothesis $D$ it is interesting to note that there was no significant difference between players of high and low participation and that age and sex of the players did not affect the results. Thus, it seems that serious games can be used to educate all parts of the population, not just the stereotypical teenage male "gamers", and that relatively little personal involvement and enthusiasm is needed for the intended message to get through. Given the diversity of play styles evident in the mass market [10], a modular design like GMP can thus conceivably serve the desired message to the greatest audience while maintaining sufficient efficacy.

In the field study, 'market penetration' was hampered by the managerial or technical barriers between the game and potential players. Pilot liaisons were also supposed to promote the game [34], but with limited resources they could not substitute for a dedicated community manager, and this led to a relatively low level of traction with regards to player recruitment and continued engagement. Nevertheless, according to the results of the field study and the 'After Green My Place' questionnaire, players enjoyed playing the game and were motivated to find out more about the themes of the mini-games via the Learn More pages. It is probable that among the dropouts player satisfaction was lower, although the collected data does not provide specific support for this claim. It is at least plausible that a serious-game-based approach was most effective among those with a mild interest in the topic, compared to those opposed to it or those who were already experts.

\section{Conclusion}

While the state of the art for serious games has moved forward since the SAVE ENERGY project, GMP still serves as a significant artifact marking (at least figuratively) a turn away from a simulationfocus and towards the new possibilities provided by the social network infrastructure of the contemporary internet world. The inconclusive quality of the hypotheses evaluated through the statistical analysis is a disappointment - it would be more satisfying to present more convincing 
supporting evidence, beyond the empirical evidence of tangible energy savings within the pilot program. Nonetheless, as a working prototype for what was, at the time, a comparatively new kind of serious game, GMP achieved some unexpected successes, and this evaluative discussion paper hopefully provides some guidance for other developers interested in a similar design.

Considering the principles given as the basis for GMP design in [4], most of them were evident in evaluation data from GMP to some degree. However, the 'affinity group' principle did not show up because there was no way to track the presence and/or importance of community in players' activity. This was due to the social infrastructure provided, and reflects in the take-home lessons.

One of the most important lessons to take from the GMP project is that the success or failure of a serious game in the SSOG format is proportional to traction. A failure to acquire, engage, and retain players means an absence of community that seriously limits what can be attained. Since the evidence of this study suggests that degree of engagement is not key a predictor of successful behavioural change, it follows that getting players involved in a serious game is potentially more important than how engaged with the game they then become or, equivalently, that community design features may be more significant contributors towards intended learning outcomes than the depth or quality of play attributable to individual game elements. The novel inclusion of real energy data from pilot buildings in GMP may thus have been more valuable for attracting players out of community interest, than for driving play (given that each player makes only a small contribution to energy consumption).

In contemporary culture, there is fierce competition for individuals' free attention. Thus, any product not intended for entertainment, such as a serious game, faces an incredible challenge to gain traction with its intended audience. Successful commercial projects provide only partial lessons because they can attract an audience by expenditure on marketing, to offset the attention problem. Serious games cannot benefit from such interventions. Questions concerning what kinds of game design are educationally effective are thus secondary to the ultimate problem facing all serious games: how can we ensure that anyone will play at all?

\section{Acknowledgement}

Responsibility for the information and views set out in this article lies entirely with the authors.

This project has been funded with support from the European Commission (grant number CIPICT-PSP-238882). This publication reflects the views only of the author, and the Commission cannot be held responsible for any use which may be made of the information contained therein.

\section{References}

[1] MacGonigal, J. (2011). Reality is broken : why games make us better and how they can change the world. London: Jonathan Cape.

[2] Gee, J. P. (2006). “Are Video Games Good for Learning?” Nordic Journal of Digital Literacy, 1(3), 172-182.

[3] Abrahamse, W., Steg, L., Vlek, C., \& Rothengatter, T. (2005). A review of intervention studies aimed at household energy conservation. Journal of Environmental Psychology, 25(3), 273291. https://doi.org/10.1016/j.jenvp.2005.08.002

[4] Cowley, B., Moutinho, J., Bateman, C., \& Oliveira, A. (2011). Learning Principles and Interaction Design for "Green My Place": a Massively Multiplayer Serious Game. Entertainment Computing, 2(2), 10. https://doi.org/10.1016/j.entcom.2011.01.001

[5] Saunders, H. D. (1992). The Khazzoom-Brookes Postulate and Neoclassical Growth. The Energy Journal, Volume 13(Number 4), 131-148. https://doi.org/10.5547/ISSN0195-6574-EJ-Vol13No4-7

[6] Michie, S., van Stralen, M. M., \& West, R. (2011). The behaviour change wheel: a new method for characterising and designing behaviour change interventions. Implementation Science : IS, 6(1), 42. https://doi.org/10.1186/1748-5908-6-42

[7] Ajzen, I. (1991). The theory of planned behavior. Organizational Behavior and Human Decision Processes, 50(2), 179-211. http://doi.org/10.1016/0749-5978(91)90020-T

[8] SAVE ENERGY. (2012). SAVE ENERGY Green Paper (pp. 1-18). Retrieved from http://www.ict4saveenergy.eu/documents 
[9] European Commission. (2006). Action Plan for Energy Efficiency: Realising the Potential $\operatorname{COM}(2006) 545 . \quad$ Brussels. Retrieved from http://ec.europa.eu/energy/action_plan_energy_efficiency/doc/com_2006_0545_en.pdf

[10] Bateman, C., Lowenhaupt, R., and Nacke, L.E. (2011). "Player typology in theory and practice", Proceedings of the Digital Games Research Association, Think Design Play 2011. Utrecht, The Netherlands.

[11] Hopson, J. (2001). Gamasutra - Features - "Behavioral Game Design" [04.27.01] \%U http://www.gamasutra.com/features/20010427/hopson_01.htm.

[12] Darby, S. (2006). The Effectiveness of Feedback On Energy Consumption. A Review for DEFRA of the Literature on Metering, Billing and direct Displays. Oxford: University of Oxford. Retrieved from http://www.eci.ox.ac.uk/research/energy/downloads/smart-meteringreport.pdf

[13] Ehrhardt-Martinez, K. (2008). Behavior, energy, and climate change policy directions, program innovations, and research paths. Conference on Behavior, Energy, and Climate Change, Washington, D.C., 2008

[14] Taylor, H., Hamilton, W. I., \& Vail, C. (2014). Creating a lasting commitment to sustainable living. In S. Sharples \& S. T. Shorrock (Eds.), Proceedings of the international conference on Ergonomics \& Human Factors (pp. 259-266). Southhampton: Taylor \& Francis.

[15] Backhaus, J. (2010). Intermediaries as Innovating Actors in the Transition to a Sustainable Energy System. Central European Journal of Public Policy, 4(1), 86-108. Retrieved from http://www.cejpp.eu/index.php/ojs/article/view/47

[16] Heiskanen, E., Johnson, M., Robinson, S., Vadovics, E., \& Saastamoinen, M. (2010). Lowcarbon communities as a context for individual behavioural change. Energy Policy, 38(12), 7586-7595. https://doi.org/10.1016/j.enpol.2009.07.002

[17] Stern PC (2008) in The Cambridge Handbook of Psychology and Economic Behaviour, ed Lewis A (Cambridge Univ Press, Cambridge, UK), pp 363-382. https://doi.org/10.1017/CBO9780511490118.015

[18] Orland, B., Ram, N., Lang, D., Houser, K., Kling, N., \& Coccia, M. (2014). Saving energy in an office environment: A serious game intervention. Energy and Buildings, 74(Supplement C), 43-52. http://doi.org/https://doi.org/10.1016/j.enbuild.2014.01.036

[19] Geelen, DV, Keyson, DV, Boess, SU \& Brezet, JC (2012). Exploring the use of a game to stimulate energy saving in households. Journal of Design Research, 10(1-2), 102-120.

[20] Fell, M. J., \& Chiu, L. F. (2014). Children, parents and home energy use: Exploring motivations and limits to energy demand reduction. Energy Policy, 65(Supplement C), 351-358. http://doi.org/https://doi.org/10.1016/j.enpol.2013.10.003

[21] Abrahamse, W., Steg, L., Vlek, C., \& Rothengatter, T. (2007). The effect of tailored information, goal setting, and tailored feedback on household energy use, energy-related behaviors, and behavioral antecedents. Journal of Environmental Psychology, 27(4), 265-276. https://doi.org/10.1016/j.jenvp.2007.08.002

[22] Petersen, J. E., Shunturov, V., Janda, K., Platt, G., \& Weinberger, K. (2007). Dormitory residents reduce electricity consumption when exposed to real-time visual feedback and incentives. International Journal of Sustainability in Higher Education, 8(1), 18. https://doi.org/10.1108/14676370710717562

[23] Lazzaro, N 2009, “Creating an MSO: Viral Emotions and the Keys to Social Play”, Presentation at Game Developers Conference 2009, San Jose CA. Available at http://www.slideshare.net/NicoleLazzaro/gdc09-mso-slides-100n032609

[24] Cowley, B. (2014). The QUARTIC Process Model for Developing Serious Games: "Green My Place" Case Study. In N. Lee (Ed.), Digital Da Vinci: Computers in the Arts and Sciences (1st ed., pp. 143-172). New York: Springer Science+Business Media.

[25] Garfield, R. (2000). "Metagames", in Dietz, J. (ed.) Horsemen of the Apocalypse: Essays on Roleplaying, Sigel, IL: Jolly Roger Games, pp. 16-22.

[26] Nintendo R\&D1 (2003). WarioWare [Videogame], Kyoto: Nintendo.

[27] Assembly Line, The (1989). Pipe Mania [Videogame], San Francisco, CA: LucasArts Entertainment.

[28] Taito (1983). Elevator Action [Arcade game], Tokyo: Taito.

[29] DMA Design (1991). Lemmings [Videogame], Liverpool: Psygnosis.

[30] Nintendo SPD (2005). Brain Training: How Old Is Your Brain? [Videogame], Kyoto: Nintendo.

[31] Nintendo EAD (2005). Big Brain Academy [Videogame], Kyoto: Nintendo.

[32] Schultz, W. P. (2001). The structure of environmental concern: concern for self, other people, and the biosphere. Journal of Environmental Psychology, 21(4), 327-339. Retrieved from 
http://www.sciencedirect.com/science/article/pii/S0272494401902270

https://doi.org/10.1006/jevp.2001.0227

[33] Davidov, E., Schmidt, P., \& Schwartz, S. H. (2008). Bringing Values Back In: The Adequacy of the European Social Survey to Measure Values in 20 Countries. Public Opinion Quarterly, 72(3), 420-445. https://doi.org/10.1093/poq/nfn035

[34] Cowley, B., Bedek, M., Heikura, T., Ribiero, C., \& Petersen, S. (2012). The QUARTIC Process Model to Support Serious Games Development for Contextualized Competence-Based Learning and Assessment. In M.-M. Cruz-Cunha (Ed.), Handbook of Research on Serious Games as Educational, Business and Research Tools: Development and Design (pp. 491-519). New York: IGI Global. https://doi.org/10.4018/978-1-4666-0149-9.ch025 